\title{
JAHN-TELLER CENTER AS QUASIPARTICLE OF FRACTIONAL STATISTICS
}

\author{
A.L. SoBolEWSKI \\ Institute of Physics, Polish Academy of Sciences \\ Al. Lotników 32/46, 02-668 Warszawa, Poland
}

(Received April 28, 1995)

\begin{abstract}
Arguments are presented towards the identification of a molecular center undergoing a linear $E \otimes e$ Jahn-Teller effect as a quasiparticle of fractional statistics. The relevance of Jahn-Teller quasiparticles for the description of the type-II superconductivity is briefly discussed.

PACS numbers: $31.10 .+z, 75.20 . \mathrm{Hr}$
\end{abstract}

\section{Introduction}

The Jahn-Teller (JT) effect [1] describes the interaction between the two components of a degenerate electronic state $(E)$ through a, usually degenerate, pair of nuclear modes $(e)$. An unusual aspect of the $E \otimes e$-type JT effect is that the Born-Oppenheimer electronic wave functions, which diagonalize the electronic Hamiltonian in the fixed-nuclei approximation, undergo a sign change when the nuclear coordinates traverse a closed path around the conical intersection [2-4]. As it has been shown by Mead and Truhlar [5], the resulting double-valuedness of the electronic wave functions can be removed, but only at the expense of introducing a vector-potential-like term into the effective Hamiltonian for the nuclear motion, which has been termed the molecular Aharonov-Bohm effect [6].

The above-mentioned sign change is a special case of Berry's geometrical phase [7] which holds implications for topics as diverse as the theory of the fractional quantum Hall effect [8], the optical activity of a helical fiber [9], superconductivity [10], and gauge field theories [11]. A more general discussion in the context of molecular physics can be found in the review article by Mead [12].

In the present paper we argue that JT centers can, in some applications, be considered as quasiparticles of fractional statistics. Thus in Sec. 2 a brief re-derivation of the adiabatic analytical solution of the linear $E \otimes e$-type Hamiltonian is presented and some of its properties are discussed. The arguments in favor of the fractional nature of the statistics of the JT quasiparticles and their relevance for description of the type-II superconductivity are discussed in Sec. 3. Some conclusions concerning high- $T_{\mathrm{c}}$ superconductors are presented in Sec. 4. 


\section{The linear $E \otimes e$-type Jahn-Teller system}

\subsection{An adiabatic solution}

In the following presentation we follow approach of Bersuker and Polinger [13]. The vibrational Hamiltonian of the linear $E \otimes e$-type JT system (i.e. without considering the quadratic terms of the vibronic interactions) is usually written in the form

$$
\hat{H}=\frac{1}{2}\left[P_{\theta}^{2}+P_{\varepsilon}^{2}+\omega_{0}^{2}\left(Q_{\theta}^{2}+Q_{\varepsilon}^{2}\right)\right]+\kappa\left(Q_{\theta} \hat{\sigma}_{x}+Q_{\varepsilon} \hat{\sigma}_{y}\right) .
$$

The first term represents the kinetic and potential energies of two-dimensional harmonic oscillator. The second term represents a linear coupling between the vibrational modes and a pair of degenerate electronic states $\left[\psi_{\theta}, \psi_{\varepsilon}\right]$, and $\hat{\sigma}_{x}$ and $\hat{\sigma}_{y}$ are Pauli matrices. The electronic eigenfunctions $\psi_{\theta}$ and $\psi_{\varepsilon}$ are often described by two-component vectors (spinors)

$$
\psi_{\theta}=\left(\begin{array}{l}
1 \\
0
\end{array}\right), \quad \psi_{\varepsilon}=\left(\begin{array}{l}
0 \\
1
\end{array}\right),
$$

i.e. they can formally be regarded as two different states of the same particle which differ in the value of the projection of the vector $\frac{1}{2} \hat{\sigma}$, the latter being analogous (in its properties) to the spin $1 / 2$ vector. This quantity, usually called the "energy spin" or pseudo-spin [14], is a vector in some auxiliary "energy" space. The projection of the energy spin on the $z$-axis has only two values $\pm 1 / 2$.

In the absence of vibronic interaction the symmetry of the $E \otimes e$-type JT system is described at least by the group $\mathrm{SU}(2) \times \mathrm{SU}(2)$. Taking into account the linear vibronic interaction, the symmetry of the system is lowered from the six-parameter group $\mathrm{SU}(2) \times \mathrm{SU}(2)$ to the one-parameter axial group $\mathrm{O}(2)$ of two-dimensional rotations [15]. An arbitrary element of this group has the form

$$
\hat{G}(\alpha)=\mathrm{e}^{\mathrm{i} \alpha\left(\boldsymbol{L}_{z}+\frac{1}{2} \hat{\sigma}_{x}\right)},
$$

where $\boldsymbol{L}_{z}$ is vibrational momentum operator, and the corresponding infinitesimal operator

$$
\hat{J}_{z}=\boldsymbol{L}_{z}+\frac{1}{2} \hat{\sigma}_{z}
$$

commutes with the Hamiltonian (1), i.e. it is an integral of motion. In the "static" limit $\left(P_{\theta}=P_{\varepsilon}=0\right)$ Hamiltonian (1), in polar coordinates $\left(Q_{\theta}=\rho \cos \varphi, Q_{\varepsilon}=\right.$ $\rho \sin \varphi)$, can be diagonalized via unitary transformation

$$
\hat{S}=\frac{1}{\sqrt{2}}\left(\begin{array}{cc}
\mathrm{e}^{-\mathrm{i} \varphi / 2} & \mathrm{e}^{-\mathrm{i} \varphi / 2} \\
\mathrm{e}^{\mathrm{i} \varphi / 2} & -\mathrm{e}^{\mathrm{i} \varphi / 2}
\end{array}\right),
$$

giving the adiabatic electronic wave functions

$$
\begin{aligned}
& \psi_{+}=\frac{1}{\sqrt{2}}\left(\mathrm{e}^{\mathrm{i} \varphi / 2} \psi_{\theta}+\mathrm{e}^{-\mathrm{i} \varphi / 2} \psi_{\varepsilon}\right), \\
& \psi_{-}=\frac{1}{\sqrt{2}}\left(\mathrm{e}^{\mathrm{i} \varphi / 2} \psi_{\theta}-\mathrm{e}^{-\mathrm{i} \varphi / 2} \psi_{\varepsilon}\right),
\end{aligned}
$$


and adiabatic energies

$$
E_{ \pm}(\rho)=\frac{1}{2} \omega_{0}^{2} \rho^{2} \pm \kappa \rho .
$$

The symmetry of the axial group $\mathrm{O}(2)$ of two-dimensional rotations is then reflected via rotational symmetry of the adiabatic potential energy surfaces of Eq. (7). The quantity $E_{\mathrm{JT}}=\kappa^{2} / 2 \omega_{0}^{2}$ is called the JT stabilization energy.

In the strong-coupling limit, i.e. for $E_{\mathrm{JT}} \gg \omega_{0}$, an analytical (approximate) solution of the vibrational Hamiltonian (1) is possible. Denoting the eigenfunctions of the Hamiltonian by $\Psi=\Phi / \sqrt{\rho}$, the Hamiltonian for $\Phi(\rho, \varphi)$ in the adiabatic basis (6) becomes

$$
\hat{S}^{+} \hat{H} \hat{S}=\left(-\frac{\hbar^{2}}{2} \frac{\partial^{2}}{\partial \rho^{2}}+\frac{1}{2} \omega_{0}^{2} \rho^{2}-\frac{\hbar^{2}}{8 \rho^{2}}\right) \hat{\sigma}_{0}+\frac{\hbar^{2}}{2 \rho^{2}}\left(L_{z}-\frac{1}{2} \hat{\sigma}_{x}\right)^{2}+\kappa \rho \hat{\sigma}_{z},
$$

where $\boldsymbol{L}_{z}=-\mathrm{i} \partial / \partial \varphi$ is the angular momentum operator. Since for the linear $E \otimes e$ JT system $\hat{J}_{z}=\boldsymbol{L}_{z}+(1 / 2) \hat{\sigma}_{z}$ is an integral of motion, and furthermore in the adiabatic basis $\hat{S}^{+} \hat{J}_{z} \hat{S}=\boldsymbol{L}_{z}$, i.e., the Hamiltonian (8) commutes with operator $\boldsymbol{L}_{z}$, then this means that the eigenfunctions $e^{\mathrm{ij} \varphi} / \sqrt{2 \pi}$ of the operator $\boldsymbol{L}_{\boldsymbol{z}}$ are also eigenfunctions of the Hamiltonian (8). In other words, the angular and radial motions can be separated and the wave function $\Phi(\rho, \varphi)$ can be taken in the form

$$
\Phi(\rho, \varphi)=\frac{1}{\sqrt{2 \pi}} \mathrm{e}^{\mathrm{i} j \varphi} \chi(\rho) .
$$

The Hamiltonian (8) for the radial $\chi(\rho)$ wave function acquires the form

$$
\hat{H}=\left(-\frac{\hbar^{2}}{2} \frac{\partial^{2}}{\partial \rho^{2}}+\frac{\hbar^{2} j^{2}}{2 \rho^{2}}+\frac{1}{2} \omega_{0}^{2} \rho^{2}\right) \hat{\sigma}_{0}-\frac{\hbar^{2} j^{2}}{2 \rho^{2}} \hat{\sigma}_{x}+\kappa \rho \hat{\sigma}_{z},
$$

where the operator $\boldsymbol{L}_{z}=\hat{S}^{+} \hat{J}_{z} \hat{S}$ has been substituted by its quantum number $j$.

On passing from (1) to (10) no additional approximations are introduced, and in this sense (10) is the exact Hamiltonian. Here the adiabatic approximation is equivalent to neglect of the term $-\hbar^{2} j^{2} \hat{\sigma}_{x} / 2 \rho^{2}$ which mixes the two sheets of the adiabatic potential energy surface. Then

$$
\hat{H}_{ \pm}=-\frac{h^{2}}{2} \frac{\partial^{2}}{\partial \rho^{2}} \pm E_{ \pm}(\rho)
$$

and

$$
E_{ \pm}(\rho)=\frac{\hbar^{2} j^{2}}{2 \rho^{2}}+\frac{1}{2} \omega_{0}^{2} \rho^{2} \pm \kappa \rho .
$$

The "dynamical" potential energy $E_{ \pm}(\rho)$ differs from the "static" one of Eq. (7) by the centrifugal energy term $\hbar^{2} j^{2} / 2 \rho^{2}$. This term is always nonzero due to semi-integer values of the quantum number $j$, the eigenvalue of the operator $\hat{J}_{z}=\boldsymbol{L}_{z}+(1 / 2) \hat{\sigma}_{z}, j= \pm \frac{1}{2}, \pm \frac{3}{2}, \pm \frac{5}{2}, \ldots$ Finally, the total adiabatic wave function of the linear $E \otimes e \mathrm{JT}$ system can be recasted in the form

$$
| \pm, n, j\rangle=\psi_{ \pm}(r, \rho, \varphi) \mathrm{e}^{\mathrm{i} j \varphi} \chi_{n}(\rho) / \sqrt{2 \pi \rho}
$$

For the lowest vibronic states localized near the bottom of the adiabatic potential $E_{-}\left(\rho_{0}\right)$ the potential energy (12) can be replaced by first terms of its power series expansion with respect to the displacement $\tau=\rho-\rho_{0}$, and one can solve the 
Schrödinger equation for $\chi(\tau)$ with Hamiltonian (11) in harmonic approximation. The energies of the vibronic states are then

$$
E_{n j}=h \omega_{E}\left(n+\frac{1}{2}\right)+\frac{\hbar^{2} j^{2}}{2 \rho_{0}^{2}}-E_{\mathrm{JT}}
$$

while the wave functions $\chi_{n}(\tau)$ are expressed as Hermite polynomials describing radial harmonic vibrations near the point $\rho_{0}$, with frequency $\omega_{E}$.

Let us notice that in the "exact" adiabatic solution sketched above, the widely celebrated gauge-structure, Berry's phase, and other peculiarities of the linear $E \otimes e$ JT problem do not appear explicitly. However, the adiabatic electronic wave functions $\psi_{ \pm}$of Eq. (6) change their sign under the rotation $\varphi \rightarrow \varphi+2 \pi$, while the total wave functions (13) remain unchanged because $j$ is semi-integer due to symmetry of the problem. On the other hand, if one curls out the change of the ign of the electronic wave functions (6) for instance by multiplying them by the phase factor $\mathrm{e}^{-\mathrm{i} \varphi / 2}$, this restores the single-valuedness, but on the expense of introducing a non-zero vector potential term in Eq. (8) by replacing $\boldsymbol{L}_{z} \rightarrow \boldsymbol{L}_{z}+\mathrm{i} \boldsymbol{A}_{\varphi}$, with $\boldsymbol{A}_{\varphi}=\frac{1}{2 \mathrm{i}} \hat{\sigma}_{0}$. The effective Hamiltonian for the motion of the nuclei thus contains a gauge-field term, the presence of which is unavoidable if the electronic eigenkets (6) are to be single valued functions of $\varphi$. If one takes the curl of the vector potential $\boldsymbol{A}_{\varphi}^{\prime}$ to find the corresponding (pseudo) magnetic field, one finds a $\delta$ function at the origin $(\rho=0)$. The non-zero diagonal vector potential $\boldsymbol{A}_{\varphi}$ of the solenoidal type is the origin of the intrinsic Aharonov-Bohm effect [6]. As it has been pointed out by Longuet-Higgins et al. [2] and by Mead [16] it leads to half-odd integral quantization numbers for pseudorotational motion of nuclei, thus restoring results of derivations $(6)-(12)$.

\subsection{Some properties of the linear JT system}

The molecular system undergoing linear $E \otimes e \mathrm{JT}$ interaction possesses rather peculiar properties. Let us first notice that similarly to the case of a normal molecule, the lowest (ground) electronic state of the JT system is accompanied by (roughly equally spaced) levels of radial harmonic vibration, and each of the vibrational levels is accompanied by a rotational fine structure (cf. Eq. (14)). But unlike the case of normal molecules, the (pseudo)rotational levels are quantized with fractional quantum numbers, and do not describe real rotations of the molecule, when all the atoms move around a common axis, but the wave of distortions when each of the atoms moves (within a proper phase) around its equilibrium position. This is illustrated in Fig. 1 for the case of a pseudorotating triatomic $X_{3}$ molecule, defining the intramolecular coordinates $Q_{\theta}$ and $Q_{\varepsilon}$ as follows:

$$
\begin{aligned}
& Q_{\theta}=\frac{1}{\sqrt{6}}\left(2 \Delta r_{12}-\Delta r_{13}-\Delta r_{23}\right), \\
& Q_{\varepsilon}=\frac{1}{\sqrt{2}}\left(\Delta r_{13}-\Delta r_{23}\right),
\end{aligned}
$$

where $\Delta r_{i j}$ denote the change of the bond length between the $i$-th and the $j$-th atoms of Fig. 1. Denoting $Q_{\theta}=\rho_{0} \cos \varphi, Q_{\varepsilon}=\rho_{0} \sin \varphi$ and transforming the atomic displacements to Cartesian coordinates one sees that when moving along the bottom of the trough (Eq. (12)) the atoms of the triatomic molecule circumscribe coherent circles (Fig. 1). The shape of the nuclear frame changes during 


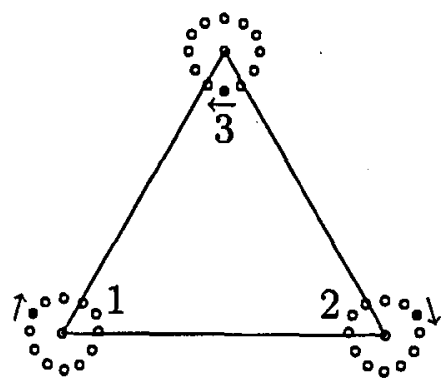

Fig: 1. Distortions of triangular molecule in the pseudorotational motion of nuclei round the trough of the lowest sheet of the adiabatic potential energy surface in the linear $E \otimes e$-type JT effect. Dots represent successive positions of nuclei shifted each other by $\Delta \varphi=30^{\circ}$ with the phase denoted by arrows. Solid dots denote position of nuclei for $\varphi=0^{\circ}$.

such a motion transforming the equilateral triangle into an isosceles one. This can be thought as a wave of distortions propagating around the geometric center of the molecule. However, the molecular pseudorotation, in fact, takes place in the space of internal molecular coordinates $Q_{\theta}$ and $Q_{\varepsilon}$.

Contrary to that, the electron density wave rotates in molecule-fixed space. In order to visualize the effect, we define the molecular orbitals $\psi_{\theta}$ and $\psi_{\varepsilon}$ in terms of a linear combination of valence atomic orbitals $\xi_{i}$ centered at the $i$-th nucleus as

$$
\begin{aligned}
& \psi_{\theta}=\frac{1}{\sqrt{3}}\left(\mathrm{e}^{\frac{2}{3} \pi \mathrm{i}} \xi_{1}+\mathrm{e}^{\frac{4}{3} \pi \mathrm{i}} \xi_{2}+\mathrm{e}^{2 \pi \mathrm{i}} \xi_{3}\right), \\
& \psi_{\varepsilon}=\frac{1}{\sqrt{3}}\left(\mathrm{e}^{-\frac{2}{3} \pi \mathrm{i}} \xi_{1}+\mathrm{e}^{-\frac{4}{3} \pi \mathrm{i}} \xi_{2}+\mathrm{e}^{-2 \pi \mathrm{i}} \xi_{3}\right) .
\end{aligned}
$$

With the aid of the transformations (6) and (16) the adiabatic electronic wave functions of the triatomic JT system can be described in terms of the atomic orbitals as

$$
\begin{aligned}
& \psi_{+}(\varphi)=\sqrt{\frac{2}{3}}\left[\cos \left(\frac{\varphi}{2}+\frac{2 \pi}{3}\right) \xi_{1}+\cos \left(\frac{\varphi}{2}+\frac{4 \pi}{3}\right) \xi_{2}+\cos \left(\frac{\varphi}{2}\right) \xi_{3}\right], \\
& \psi_{-}(\varphi)=\sqrt{\frac{2}{3}}\left[\sin \left(\frac{\varphi}{2}+\frac{2 \pi}{3}\right) \xi_{1}+\sin \left(\frac{\varphi}{2}+\frac{4 \pi}{3}\right) \xi_{2}+\sin \left(\frac{\varphi}{2}\right) \xi_{3}\right] .
\end{aligned}
$$

The variation of the LCAO coefficients vs. $\varphi$ for the $\psi_{-}$adiabatic wave function is illustrated in Fig. 2. Upon inspection of this figure, one can easily notice the change of the sign of the $\psi_{-}$-wave function after $2 \pi$ rotation in the internal coordinates space. In this simple model, which neglects the interactions of the unpaired electron of the JT center with the other electrons of the molecular system, the charge and spin densities are proportional to the square of the LCAO coefficients and it is seen that both, the charge and the spin density waves propagate around the JT center with the frequency of molecular pseudorotation. This is an explicit manifestation of the special coupling of the vibrational momentum $\boldsymbol{L}$ and the energy spin $\hat{\sigma} / 2$ due to which they are not conserved (each of them) separately. The conserved value is the projection of the total momentum $\hat{J}=\boldsymbol{L}+\frac{1}{2} \hat{\sigma}$ on the $z$-axis. Thus the wave of electron density and the wave of nuclear distortions are no longer independent, but are coherently related. The question whether rotation 


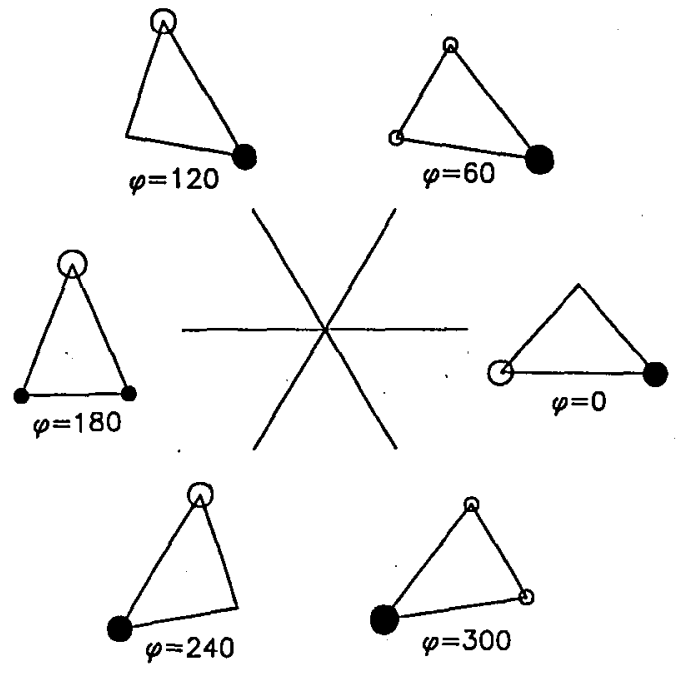

Fig. 2. Behavior of the LCAO coefficients of the $\psi_{-}$adiabatic molecular orbital of the triangular molecule subjecting to the linear $E \otimes e$-type JT effect when the nuclei are taken round the trough of the lowest sheet of the adiabatic potential as illustrated in Fig. 1. The marked value of the rotational angle $\varphi$ is in degrees. The size of dots is proportional to the magnitude of the LCAO coefficients, while the negative sign is marked by solid dots.

of the electron density around the JT center is caused by a gauge potential, or whether the solenoidal magnetic field at the center results from the rotation of electron density is somewhat a semantic one. This directly relates to the "reduced" approach used in the mathematical description of the system in which variables of one of the subsystems are integrated out.

Let us finally notice that the solenoidal-like magnetic field at the JT center is not a fictitious one, but it is a real magnetic field related to the rotation of the electron density wave around the JT center. This can easily be shown by calculating the expectation value of the magnetic dipole operator for the wave function given by Eq. (13). Since the eigenfunctions of the operator $\boldsymbol{L}_{\boldsymbol{z}}$ are also eigenfunctions of the total vibrational Hamiltonian (Eq. (8)) the magnitude of the magnetic dipole moment at the JT center is proportional to the rotational quantum number $j$ of its given eigenstate. This can never be zero because $j \neq 0$. In other words, the linear $E \otimes e \mathrm{JT}$ center is a source of a real permanent magnetic field of a solenoidal type. This conclusion remains true also for higher (quadratic) order coupling as long as the quadratic coupling does not destroy the pseudorotational motion of the system.

\section{Discussion}

An important and unique feature of the JT system resulting from the non-separability of the electronic and nuclear motion is that it presents itself as a composite object (a quasiparticle) consisting of a boson (a deformable nuclear 
frame) and a fermion (rotating electronic density). Such quasiparticles can be thought of as they were bosons carrying a magnetic solenoid containing a fraction $\nu(\nu= \pm 1 / 2$ for the ground state) of a flux quantum. Thus there is a long-range magnetic dipole-dipole interaction between two JT centers. Moreover, in the "reduced" description, where the intramolecular degrees of freedom are integrated out, the magnetic flux of the JT quasiparticle can be viewed as resulting from the underlying gauge structure of the vector potential. The wave function of the system of such quasiparticles thus acquires a phase $\mathrm{e}^{\mathrm{i} \nu \pi}$ when two particles are adiabatically interchanged.

The gas of quasiparticles obeying $\nu=1 / 2$ fractional statistics possesses rather peculiar features since it is somewhat between bosons $(\nu=0)$ and fermions $(\nu=1)$. Its properties have been extensively investigated over the recent years with particular respect to the fractional quantum Hall effect $[8,17]$ and to the novel class of the high- $T_{\mathrm{c}}$ superconductors $[10,18,19]$. Although particles obeying $\frac{1}{2}$-fractional statistics are not bosons, and cannot show Bose condensation, pairs of them, like pairs of electrons, are bosons and can Bose condense. Moreover, in 2+1 dimensions particles obeying $\nu$-fractional statistics interact with each other via a particular long-range potential resulting from the "statistical" gauge field. The presence of long-range potentials causes the excitations from the ground state of such systems to have a finite energy gap $[18,19]$. In other words, the ground state of such system, the spin-liquid state, may serve as a basic model for the type-II superconductors.

The discussion presented above explicitly shows that one can rather convincingly identify the linear $E \otimes e \mathrm{JT}$ system in its ground state as a quasiparticle obeying $\frac{1}{2}$-fractional statistics. Moreover, in a molecular layer of JT quasiparticles constructed in such a way that it preserves the symmetry for a coherent JT effect (for instance, in the case of $C_{3}$ local symmetry point group, each of the JT centers must have three nearest-neighbors, and form a kind of the kagome or the 3-12 lattices $[20,21])$ the unique dynamics of the $\frac{1}{2}$-fractional statistics can be preserved. The wave of molecular distortions of the lattice of the JT centers is of a special kind. It consists of coherent pseudorotations of each of quasiparticles with momentum and energy spin of opposite sign on the nearest-neighboring sites. Since each of the JT centers contains an unpaired electron, at any instant of time they form a layer of molecular dimers with common pair of electrons, a kind of Mott insulator. An important difference between the layer of the JT quasiparticle dimers and a Mott insulator is the dynamics inherent in the former. The pairs of electrons which bond a particular molecular dimer do not reside on a "static" local molecular orbital. In fact, a particular JT quasiparticle forms and breaks the bonds with its successive neighbors, due to the zero-energy pseudorotational motion of the electron density wave (cf. Fig. 2), analogous to the spin-liquid state [22]. In each of the temporary formed dimers, bonding electrons lose their identity and thus the electric current is generated in any external electric field lying in the plane of the layer. At sufficiently low temperatures which do not destroy a long-range order between quasiparticles, this should be the superconductive current of the type-II superconduictor.

As was recently pointed by Kivelson and Rohksar [23], fractional statistics 
and thus the mechanism of superconductivity outlined above, makes no sense in system with time-reversal invariance. It has subsequently been shown by Wu et al. [24] that tactics in the theory is spontaneous breaking of time-reversal and parity invariance. In other words, there are two so-called spin-liquid states related to one another by complex conjugation, and the system must choose between them when condenses into the superconducting state.

\section{Conclusions}

The mechanism of the coherent pseudorotations of molecular distortions within a layer of JT centers coupled with the coherent (real-space) rotations of the electron density as sketched above may provide an attractive description of the type-II superconductivity. Indeed, there are strong indications that the JT centers play a crucial role in the new class of high- $T_{\mathrm{c}}$ (HTC) superconductors. Both widely investigated classes of the HTC materials, the copper oxides [25, 26] and fullerenes [27-29], contain JT centers (see also [30] and references therein). This would confirm the expectation of Bednorz and Müller expressed in the words: "The guiding idea in developing the concept was influenced by the Jahn-Teller polaron model..." [31]. Let us stress, however, that there is a fundamental difference between the JT quasiparticle model proposed here and the JT based polaronic [32] or bipolaronic [33] models which are often quoted in literature. A polaron, by its definition, denotes a wave of distortions propagating through the lattice and coherently interacting with the electronic density wave. In other words, it represents a version of the BCS classical model. The "polarons" in the JT quasiparticle model are localized on particular pseudorotating JT centers. The phases of the pseudorotations between different JT quasiparticles are correlated due to the long-range gauge (or magnetic dipole-dipole) interactions forming a spin-liquid state. If the pseudorotational (zero-energy) motion is hindered due to symmetry reasons or due to strong second-order vibronic interactions, the system is expected to be a kind of Mott insulator.

The problems connected to the construction of the JT quasiparticle layer and its properties are discussed in more detail in the forthcoming paper [34].

\section{Acknowledgments}

I thank Prof. W. Domcke for stimulating discussions during the course of this study.

\section{References}

[1] H.A. Jahn, E. Teller, Proc. R. Soc. A 161, 220 (1937).

[2] H.C. Longuet-Higgins, U. Opick, M.H.L. Pryce, R.A. Sack, Proc. R. Soc. Lond. A 244, 1 (1958).

[3] G. Herzberg, H.C. Longuet-Higgins, Discuss. Faraday Soc. 35, 77 (1963).

[4] M.C.M. O'Brien, Proc. R. Soc. Lond. A 281, 323 (1964). 
[5] C.A. Mead, D.G. Truhlar, J. Chem. Phys. 70, 2284 (1979).

[6] Y. Aharonov, D. Bohm, Phys. Rev. 115, 485 (1959).

[7] M.V. Berry, Proc. R. Soc. Lond. A 392, 45 (1984).

[8] D. Arovas, J.R. Schrieffer, F. Wilczek, Phys. Rev. Lett. 53, 722 (1984).

[9] R.Y. Chiao, Y.-S. Wu, Phys. Rev. Lett. 57, 933 (1986).

[10] R.B. Laughlin, in: Mechanisms of High Temperature Superconductivity, Eds. H. Kamimura, A. Oshiyama, Springer Series in Material Sciences, Vol. 11, Springer, Berlin 1989, p. 76.

[11] F. Wilczek, A. Zee, Phys. Rev. Lett. 52, 2111 (1984).

[12] C.A. Mead, Rev. Mod. Phys. 64, 51 (1992).

[13] I.B. Bersuker, V.Z. Polinger, Vibronic Interactions in Molecules and Crystals, Springer Series in Chemical Physics, Vol. 49, Springer, Berlin 1989.

[14] R.P. Feynman, F.L. Vernon, P.W. Helwarth, J. Appl. Phys. 28, 49 (1957).

[15] C.H. Leung, W.H. Kleiner, Phys. Rev. B 10, 4434 (1974).

[16] C.A. Mead, Chem. Phys. 49, 23 (1980).

[17] B.I. Halperin, Phys. Rev. Lett. 52, 1583 (1984).

[18] R.B. Laughlin, Phys. Rev. Lett. 60, 2677 (1988).

[19] J.L. Levy, R.B. Laughlin, Phys. Rev. B 50, 7107 (1994).

[20] K. Yang, L.K. Warman, M.S. Grivin, Phys. Rev. Lett. 70, 2641 (1993).

[21] J.H. Barry, M. Khatun, Phys. Rev. B 51, 5840 (1995).

[22] P.W. Anderson, Science 235, 1196 (1987).

[23] S.A. Kivelson, D.S. Rohksar, Phys. Rev. Lett. 61, 2630 (1988).

[24] X.G. Wu, F. Wilczek, A. Zee, Phys. Rev. B 39, 11413 (1989).

[25] W.E. Pickett, H. Krakauer, R.E. Cohen, D. Singh, Physica C 161/164, 1419 (1989).

[26] D.P. Clougherty, K.H. Johnson, M.E. McHenry, Physica C 162/164, 1475 (1989).

[27] K.H. Johnson, M.E. McHenry, D.P. Clougharty, Physica C 183, 299 (1991).

[28] M. Lannoo, G.A. Baraff, M. Schlüter, D. Tomanek, Phys. Rev. B 44, 12106 (1991).

[29] O. Gunnarsson, H. Handschuh, P.S. Berthold, B. Kessler, G. Ganteför, W. Eberhart, Phys. Rev. Lett. 74, 1875 (1995).

[30] M.C.M. O'Brien, C.C. Chancey, Am. J. Phys. 61, 688 (1993).

[31] J.G. Bednorz, K.A. Müller, Rev. Mod. Phys. 60, 585 (1988).

[32] P.G. de Gennes, J. Phys. Radium 23, 630 (1962).

[33] N.F. Mott, Physica A 200, 127 (1993).

[34] A.L. Sobolewski, W. Domcke, to be published. 